\title{
El impacto disruptivo del libro electrónico sobre la cadena de valor editorial española: un estudio de casos
}

\author{
Marta Magadán-Díaz*, Jesús I. Rivas-García* \\ * Universidad Internacional de La Rioja, UNIR, Logroño, La Rioja \\ Correo-e: marta.magadan@unir.net | ORCID iD: https://orcid.org/0000-0003-3178-3215 \\ Correo-e: jesus.rivas@unir.net | ORCID iD: https://orcid.org/0000-0003-0576-5961
}

Recibido: 15-02-2019; 2a versión: 29-04-2019; Aceptado: 04-05-2019

Cómo citar este artículo/Citation: Magadán-Díaz, M.; Rivas-García, J. I. (2020). El impacto disruptivo del libro electrónico sobre la cadena de valor editorial española: un estudio de casos. Revista Española de Documentación Científica, 43 (1), e258. https://doi.org/10.3989/redc.2020.1.1650

Resumen: El presente trabajo analiza el impacto disruptivo de la aparición del libro electrónico sobre la cadena de valor en la industria editorial española. Se lleva a cabo un estudio de casos de tres empresas editoriales españolas. Los principales resultados obtenidos son: a) el desarrollo del libro electrónico no incentiva a la editorial a reducir su producción de libro en papel sino que complementa dicha actividad tradicional, b) el desarrollo del libro electrónico incentiva a la editorial a redefinir su cadena de valor para integrar el nuevo producto, c) el nuevo contexto digital abre a las editoriales españolas la posibilidad de obtener ventajas derivadas de la desintermediación, y d) los modelos de negocio existentes en editoriales ya consolidadas deben ser repensados para adaptarlos al libro electrónico, lo que puede implicar una apuesta por mantener ambos formatos -papel y electrónico- o por la renuncia al papel, transitando hacia un modelo exclusivamente dedicado al libro electrónico.

Palabras clave: industria editorial; libro electrónico; cadena de valor; desintermediación; digitalización, España.

\section{Disruptive impact of e-book on Spanish publishers' value chain: a case study}

Abstract: The present work analyses the disruptive impact of the e-book on the Spanish publishers' value chain. A case study on three Spanish publishing companies is carried out. The most relevant results obtained are: a) the development of e-book does not encourage the publisher to reduce its production of paper books but complements this traditional activity, b) the development of e-book encourages the publisher to redefine its value chain to integrate the new product, c) the new digital context opens the possibility for Spanish publishers to obtain advantages derived from disintermediation, and d) existing business models in already consolidated publishing companies must be rethought to adapt them to e-book, which can be imply a commitment to maintain both formats -paper and electronic- or renounce paper and move towards a business model solely dedicated to e-books.

Keywords: publishing industry; e-book; value chain; disintermediation; digitization, Spain.

Copyright: () 2020 CSIC. Este es un artículo de acceso abierto distribuido bajo los términos de la licencia de uso y distribución Creative Commons Reconocimiento 4.0 Internacional (CC BY 4.0). 


\section{INTRODUCCIÓN}

A comienzos del siglo XXI, las editoriales españolas comenzaban a publicar libros electrónicos $y$, al mismo tiempo, las bibliotecas públicas daban sus primeros pasos hacia la transformación digital experimentando con los préstamos de libros electrónicos (Sánchez y otros, 2011).

Algunas iniciativas a nivel internacional, como NetLibrary (de la cadena de librerías Barnes and Noble) o e-publishing (de Random House), entre otras, se lanzaron a comercializar libros electrónicos, alentadas por predicciones que estimaban que el mercado de libros electrónicos dominaría hasta un tercio de la industria editorial. Sin embargo, muchas de ellas fracasaron debido, entre otros factores, a la escasez de contenidos digitalizados, a la incompatibilidad de dispositivos y al desconocimiento del uso de dichas tecnologías por parte de los potenciales consumidores (Humphreys, 2006).

Será con el lanzamiento del Kindle (de Amazon) y del iPad (de Apple) cuando comience a desarrollarse la distribución y comercialización del libro electrónico (Lin y otros 2013), lo que sugiere que uno de los factores limitantes principales para el desarrollo de la industria editorial digital fue la falta de un lector electrónico suficientemente sofisticado como para atraer a los lectores al ámbito digital (Gaigher, 2012).

Solo en 2017, se lanzaron 28.433 nuevos títulos de libros electrónicos en España, lo que representa casi el $30 \%$ de la producción editorial (Magadán y Rivas, 2018b). La facturación asociada a la venta de libros electrónicos creció un $11 \%$ en 2016 y un 14\% en 2017 (Observatorio de la Lectura y el Libro, 2018). Sin embargo, la contribución de los libros electrónicos a los beneficios totales de las principales editoriales del país sigue siendo insignificante, apenas llega al 5\% de la facturación total, en 2017 (Observatorio de la Lectura y el Libro, 2018).

La irrupción de las tecnologías de la información y de la comunicación ha diversificado y ampliado los canales de distribución y difusión de productos editoriales. Hasta hace pocos años, la estructura de distribución editorial era el resultado de una forma de hacer específica del sector (Magadán, 2017). Sin embargo, con la aparición disruptiva del libro electrónico, la industria editorial española está viviendo una importante transformación (Carreiro, 2010; Magadán y Rivas, 2018a), surgiendo nuevas configuraciones en la cadena de suministro de libros y dando lugar a la necesidad de estudios sobre su impacto y estrategias de gestión.

El objetivo general de este trabajo es analizar el impacto que los libros electrónicos tienen sobre los procesos internos en la cadena de valor de las edi- toriales españolas, al que se añaden dos objetivos más específicos: a) estudiar la nueva configuración de la cadena de valor ampliada del sector editorial, y b) identificar las estrategias editoriales de adaptación a los intereses de la demanda.

La relevancia de este trabajo de investigación es que no se ha realizado un estudio de naturaleza cualitativa similar para la industria editorial española, cuyo objetivo sea abordar el impacto del libro electrónico sobre la cadena de suministro editorial, considerando el período temporal que va desde el arranque de la crisis económica (2007) hasta el último año del que se tienen datos de referencia en el momento del desarrollo de esta investigación (2018).

El trabajo está estructurado de la siguiente manera: el apartado dos proporciona un marco teórico para esta investigación; el tercer apartado describe el método aplicado y presenta el estudio de casos; el apartado cuatro discute los hallazgos obtenidos; y el apartado cinco resume las principales conclusiones.

\section{MARCO TEÓRICO}

A continuación, se expondrán aquellas teorías relevantes sobre la cadena de valor editorial y el libro electrónico, que constituirán el marco conceptual para el estudio de casos en esta investigación.

\section{La cadena de valor editorial}

El análisis de la cadena de valor editorial es uno de los medios fiables para entender su estructura y funciones (Bhatiasevi y Dutot, 2014). Por otra parte, la era digital impacta sobre los modelos de negocios (Magadán y Rivas, 2018a) y las cadenas de valor tradicionales, reduciendo las barreras de entrada a nuevos operadores -como los proveedores tecnológicos- (Benghozi y Salvador, 2015) y otorgando un papel destacado a los nuevos canales de distribución (Benhamou, 2015).

En la figura 1 se describen la red de relaciones e interacciones existentes entre el editor y el resto de agentes directa e indirectamente vinculados a la industria del libro en papel.

Para derivar la red de relaciones existentes en la cadena de valor del libro en papel es necesario considerar como criterio de selección de los eslabones el relativo a la relación directa con el editor en el proceso de identificación de la propuesta de valor (obra), su producción y comercialización física. Con la identificación de la propuesta de valor, tendremos al autor y a los agentes; con la producción, nos encontramos con los impresores y, finalmente con la comercialización, identificamos a los distribuidores -en un sentido amplio que englobe, tam- 
bién a los exportadores- y librerías. Finalmente, el lector-cliente es el destinatario de la acción empresarial editorial, que cerraría la cadena de valor.

A partir de lo indicado anteriormente, se muestra en la figura 2 el conjunto de eslabones clave que conforman la cadena de valor tradicional en el sector editorial y cómo se establecen las relaciones entre ellos (Magadán, 2017).

El autor escribe un original que remite -directamente o por mediación del agente- al editor. Posteriormente, el editor transforma el manuscrito recibido en un libro en papel producido en una imprenta y se lo entrega al distribuidor, que lo promociona y lo coloca en las librerías. Por último, los libreros exhiben, prescriben y venden el producto editorial al cliente/lector.
El editor es el eslabón nuclear en esta cadena al asumir los riesgos financieros de la producción del libro y al fijar su precio de venta. Sin embargo, con la llegada de Internet y del libro electrónico, comienza un proceso que podría definirse como de desintermediación: a) se reduce el número de intermediarios en las transacciones y el flujo de información (Caputo, 2012), y b) aumentan las transacciones vía canales directos, es decir, con ausencia total de intermediarios (Marszałek, 2016).

La desintermediación del sector permite a las editoriales comercializar sus libros de manera independiente, ya sea: a) sin tener en cuenta a la librería, a través de ventas directas en sitios web; b) sin tener en cuenta al distribuidor, comercializando su fondo directamente con la librería, o c) sin tener en cuen-

Figura 1. Interacción de la empresa editorial (libro en papel) con otros sectores

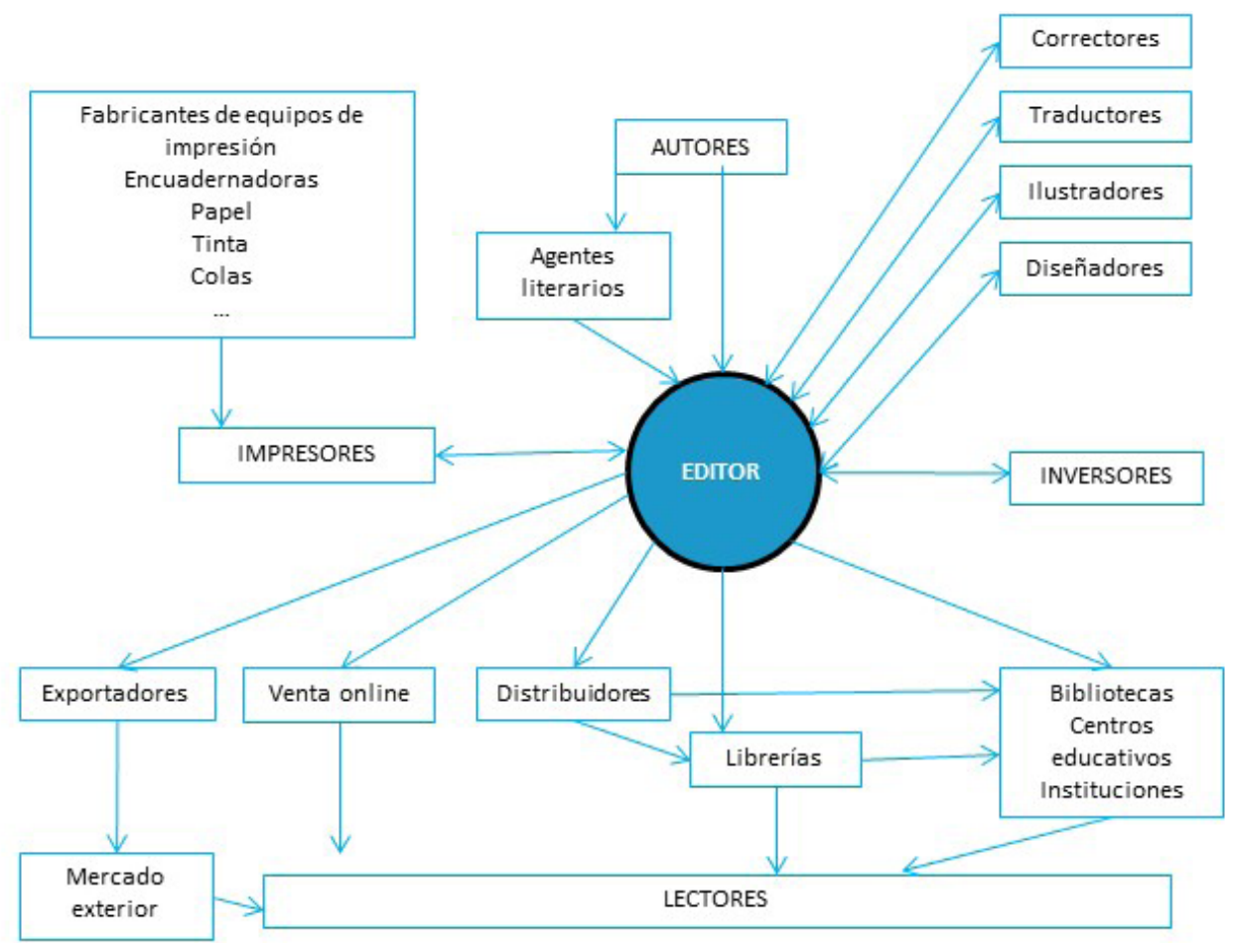

Figura 2. La cadena de valor tradicional del sector editorial

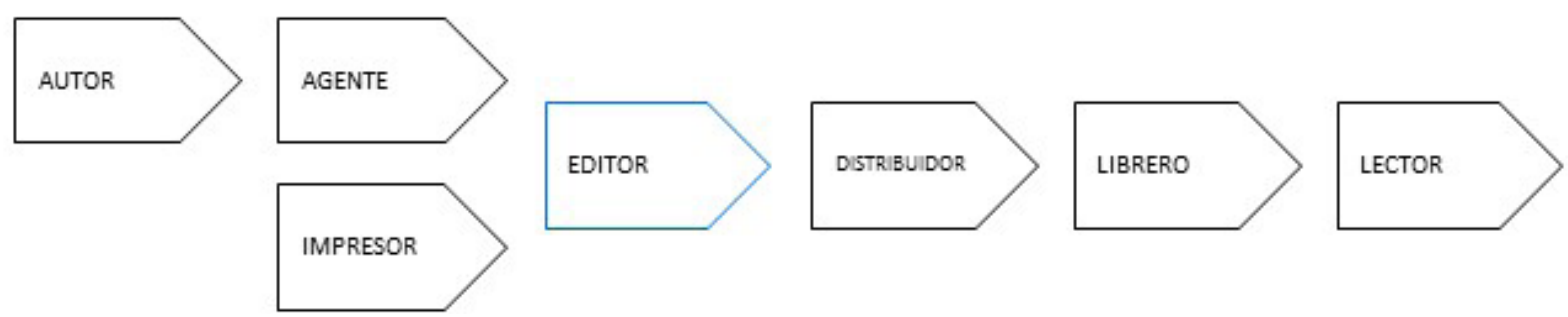

Fuente: Magadán (2017, pág. 172) 
ta a ninguno de los dos eslabones, librería y distribuidor, asumiendo tales funciones en su modelo de negocio. Sin embargo, inherente a este escenario es también el hecho de que los editores van perdiendo progresivamente el control sobre el negocio editorial e, incluso, pueden llegar a ser un eslabón cada vez menos necesario si los autores optan por asumir todo el proceso de publicación en el nuevo contexto digital (Brooks y Fitz, 2015; Gaigher, 2012).

Tradicionalmente, el fenómeno de la autopublicación era muy poco significativo estadísticamente, dados los elevados costes que un particular -en este caso, potencial autor- debería asumir, lo que hacía poco accesible esta opción, concentrándose en el sector editorial el poder decisorio sobre lo que se publicaba o no (Magadán, 2017). Sin embargo, con la irrupción del libro electrónico se desplaza ese poder decisorio y se facilita el acceso a la condición de autor a través de dicha autopublicación (Alonso y Cordón, 2011).

Además de potenciar la autopublicación, el libro electrónico quiebra, por así decirlo, el vínculo entre edición y tirada, liberando a la primera de un ancla definido como "tirada mínima" que, en el caso del libro en papel, era de obligada producción para poder recuperar, al menos, la inversión inicial. $Y$ en todo ese movimiento "liberador" el nuevo formato digital elimina de la estructura de costes los relativos a impresión, almacenamiento y logística de distribución física (Matulionyte y otros, 2017).

Pero dicha disrupción va un paso más allá derribando fronteras y límites físicos para editoriales de menor tamaño con dificultades de acceso físico a determinados mercados. Internet y el formato digital, cambian completamente el escenario y las reglas del juego en el negocio editorial (Alonso y Cordón, 2011; Magadán, 2017).

En definitiva, la digitalización en el sector editorial aporta nuevas formas de presentar el contenido (Shatzkin, 2008) y permite que se puedan desarrollar nuevos modelos de negocio (Magadán y Rivas, 2018a), como la autopublicación (Peng, 2016).

\section{El libro electrónico}

En un sentido seminal, el libro es el material de construcción básico del conocimiento, la cultura y la información (Magadán, 2017). Por tanto, la esencia del libro radicaría en su fondo y no en su forma (Peng, 2016).

Existen múltiples definiciones de libro electrónico (Rao, 2005; Berube, 2005). Un libro electrónico puede considerarse: a) una versión digitalizada de un libro en formato papel para visionarlo sobre un dispositivo electrónico determinado (Orosa y García, 2016), b) cualquier obra legible en pantalla (Carreiro, 2010), c) el dispositivo lector en sí mismo (Carreiro, 2010), y d) cualquier archivo digitalizado susceptible de descarga y decodificación en dispositivos electrónicos de lectura y visualización (Vasileiou y Rowley, 2008).

En resumen, un libro electrónico es un archivo digital que requiere de dos elementos integrados para poder ser decodificado: un dispositivo físico de lectura -hardware- y un programa decodificador -software- capaz de interpretar el contenido del archivo (Gaigher, 2012).

En el caso de España, la edición de libros electrónicos experimentó en los últimos años un aumento significativo. En la figura 3 se muestran los datos relativos a los libros electrónicos publicados entre 2007 y 2017 en España, así como su tendencia a través del método de las medias móviles.

Los datos mostrados en la figura 3 subrayan el modo en que el comienzo de la crisis financiera de 2008 impulsa en la industria editorial española un movimiento estratégico hacia la edición de libros electrónicos con un crecimiento sostenido entre 2008 y 2011 que se va desacelerando entre 2011 y 2013, para estancarse entre 2013 y 2015 y volviendo a despegar a partir de ese mismo año con motivo de una moderada recuperación económica.

La figura 4 recoge la facturación asociada al libro electrónico en España, expresada en millones de euros.

La facturación de los libros electrónicos en España superó los 119 millones de euros en 2017, lo que significó un incremento de aproximadamente casi dos millones con respecto a la cifra registrada el año anterior, aunque no va más allá del $5 \%$ de la cifra de negocio global de las editoriales españolas (Observatorio de la Lectura y el Libro, 2018).

Algunos de los factores responsables de la baja facturación del libro electrónico en España son: a) el precio elevado del libro electrónico, que casi siempre es cercano o incluso superior al del libro impreso, como consecuencia, principalmente, del diferente tratamiento fiscal del libro electrónico respecto al libro en papel (Magadan y Rivas, 2019), b) el precio elevado de los e-readers con una relación coste-beneficio poco competitiva (Díaz, 2010), c) la incompatibilidad entre las diferentes plataformas de lectura (Chao y otros, 2013), d) el estricto control de los derechos de autor (Alonso y Cordón, 2011), e) la existencia de plataformas de descarga ilegal (Jonas y Sirkeci, 2018) y f) la falta de integración entre los proveedores de contenido (Chao y Lu, 2011).

El libro electrónico ha modificado el canal de distribución tradicional, lo que impacta directamente sobre distribuidores y librerías independientes, 
Figura 3. Libros electrónicos publicados en España (2007-2017)

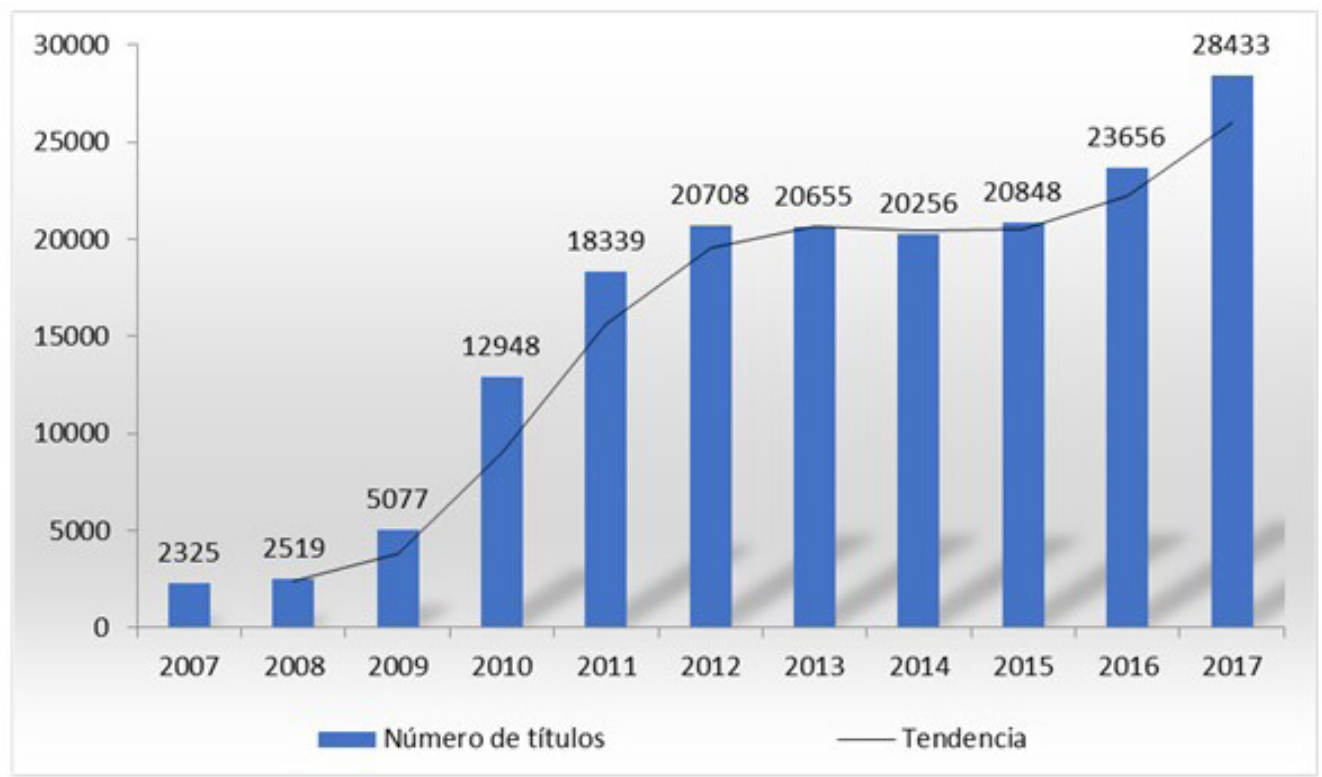

Fuente: Panorámica de la Edición Española de Libros.

Figura 4. Cifra de negocio del libro electrónico en España (2007 a 2017) (en millones de euros)

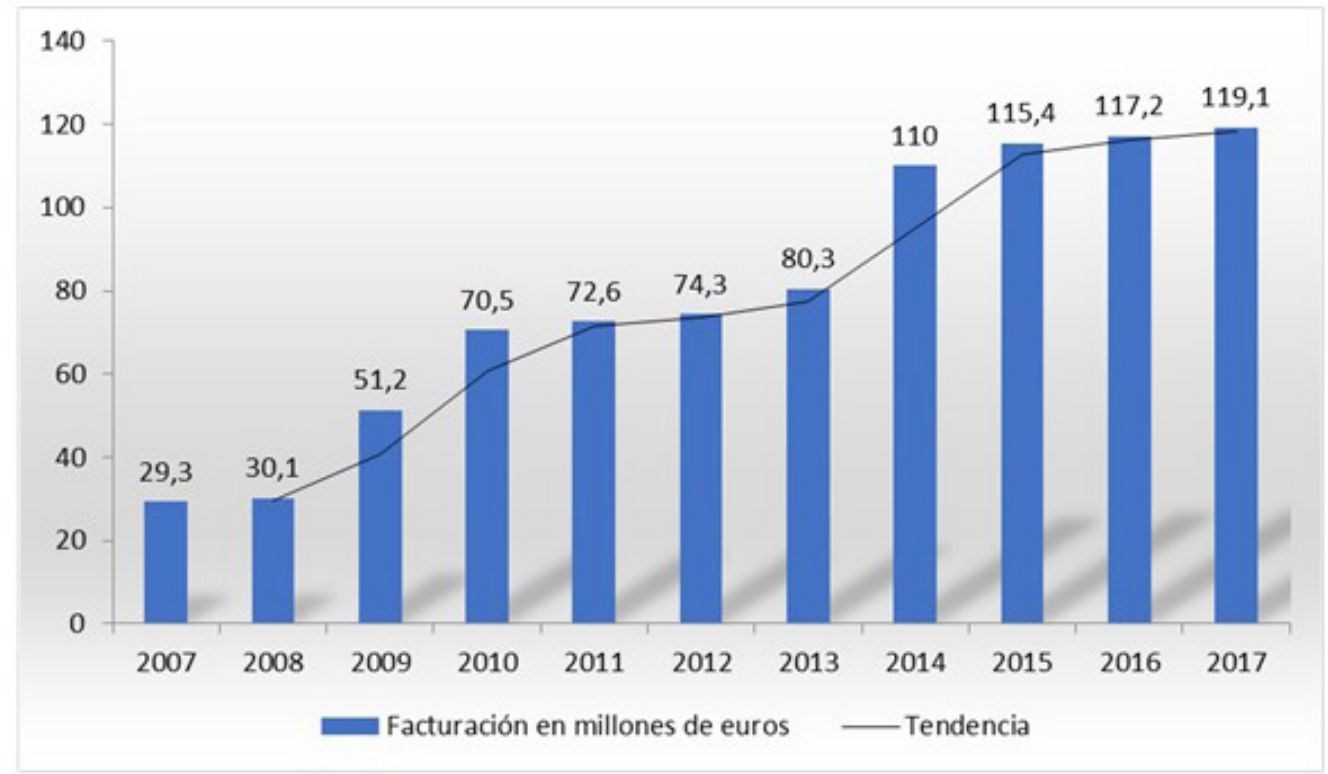

Fuente: El Comercio Interior del Libro (FGEE, 2007-2017).

que no podrán competir con los nuevos operadores quienes, lejos de limitarse a la oferta editorial tradicional, ofrecen una nueva intermediación que altera los patrones existentes de distribución y las políticas de fijación de precios (Longhi y Rochhia, 2014; Magadán, 2017).
Finalmente, la edición de libro electrónico exige costes de aprendizaje y adaptación a los nuevos formatos y fuertes inversiones en tecnología que eviten la piratería y preserven la propiedad intelectual, costes que son difíciles de superar para una pequeña o mediana empresa (Magadán, 2017). 


\section{METODOLOGÍA}

Este trabajo pretende comprender el impacto de los libros electrónicos en la dinámica y la estructura de la cadena de valor editorial en España. La metodología empleada para realizar el estudio empírico es la del método del estudio de casos. Este enfoque es de naturaleza fundamentalmente interpretativa (Cresswell, 2003) y se ajusta al alcance y objetivos de este estudio porque ofrece la posibilidad de explicar o comprender un fenómeno, un proceso o una combinación de éstos (Corbetta, 2003). De hecho, esta metodología resulta ser muy adecuada cuando las preguntas clave son qué (descripción), cómo y por qué (aplicación) en la generación de una teoría (Snow y Thomas, 1994).

De la amplia literatura sobre el método de estudio de casos, sus fundamentos y aplicaciones se pueden destacar obras y estudios como los de Eisenhardt (1989), Chetty (1996), Gerring (2007), Simons (2011), Yin (2011) o Elman y otros, (2016). Dichos trabajos son considerados seminales en una aproximación actualizada a la comprensión y aplicación del método de estudio de casos.

La metodología del estudio de casos se ha venido aplicando a investigaciones de dirección de empresas sobre asociaciones y acuerdos de cooperación empresarial (Wilson y Vlosky, 1997; Yin, 2009), sobre procesos directivos y organizativos (Grunow, 1995; Mintzberg, 1973) o relativos al cambio organizativo e innovación (Brown y Eisenhardt, 1997; McCutcheon y Meredith, 1993; Pettigrew y Whipp, 1991; Van de Pen y Poole, 1990), entre otros. En este contexto, hemos optado por emplear dicha metodología para desarrollar el análisis empírico de esta investigación, ya que el estudio de casos es una de las principales estrategias de investigación dentro del campo cualitativo, especialmente con respecto a la gestión de operaciones (Ketokivi y Choi, 2014).

Para comprender las dinámicas que se han venido produciendo en la cadena de valor editorial, en lugar de considerar todos sus vínculos y eslabones, esta investigación se situó en la perspectiva de las empresas editoriales considerándolas los centros de gravedad de aquélla y otorgándoles un papel principal respecto al resto de eslabones.
Las editoriales que componen este estudio fueron seleccionadas según un criterio de conveniencia (accesibilidad y perfil buscado para el conjunto de casos); por lo tanto, dada la dificultad de acceder a profesionales en esta rama, solo se seleccionaron aquéllos que podían contribuir efectivamente a la pregunta de investigación, una condición necesaria en los estudios de casos (Cresswell, 2003; Miles, Huberman y Saldaña, 2013).

En esta investigación se han utilizado distintos métodos de recogida de evidencias: búsqueda y revisión de documentos de las empresas analizadas, realización de entrevistas estructuradas y, en su caso, grabación en audio. Esto responde a la necesidad metodológica de una triangulación informativa que evalúe el alineamiento de las respuestas obtenidas de las editoriales a las evidencias documentales existentes y disponibles públicamente.

Las empresas seleccionadas (muestreo por conveniencia), fueron tres editoriales, cuyos nombres reales fueron omitidos: una de gran tamaño (editorial E1), una de tamaño mediano (editorial E2) y una de tamaño pequeño (E3). Con objeto de evitar que una ubicación precisa pudiera dar lugar a una identificación de la organización entrevistada, para preservar su anonimato se ha dividido el territorio nacional en cinco áreas: a) Norte (Galicia, Asturias, Cantabria, País Vasco, La Rioja, Navarra y Aragón); b) Mediterráneo (Cataluña, Valencia y Murcia); c) Sur (Andalucía y Extremadura); d) Centro (Castilla-León, Madrid y Castilla-La Mancha); y e) Islas y Ciudades Autónomas.

En la tabla I se muestra de modo resumido el perfil de cada una de las editoriales estudiadas (año de creación, forma jurídica, rango de empleados, rango de facturación, sector de actividad y ubicación geográfica).

Se realizaron tres entrevistas de octubre a noviembre de 2018, con un cada representante de cada una de las tres editoriales seleccionadas. Las entrevistas fueron realizadas por los propios autores del estudio; una fue en persona y el resto fue por correo skype. Hemos pretendido que las preguntas fuesen lo suficientemente amplias para que los responsables entrevistados pudieran profundi-

Tabla I. Descripción de los casos a estudiar

\begin{tabular}{|c|c|c|c|c|c|c|c|}
\hline \multirow{2}{*}{ EMPRESAS } & $\begin{array}{c}\text { DENOMI- } \\
\text { NACIÓN }\end{array}$ & $\begin{array}{c}\text { ANTIGUE- } \\
\text { DAD }\end{array}$ & $\begin{array}{c}\text { FORMA } \\
\text { JURIDICA }\end{array}$ & $\begin{array}{c}\text { RANGO DE } \\
\text { EMPLEA- } \\
\text { DOS }\end{array}$ & $\begin{array}{c}\text { RANGO DE } \\
\text { FACTURA- } \\
\text { CIÓN (en } \\
\text { millones de } \\
\text { euros) }\end{array}$ & $\begin{array}{c}\text { SECTOR DE } \\
\text { ACTIVIDAD }\end{array}$ & UBICACIÓN \\
\hline \multirow{3}{*}{ Editoriales } & E1 & 58 & S.A. & $>250$ & $>60$ & 5811 & Centro \\
\cline { 2 - 8 } & E2 & 25 & S.L. & $5-25$ & $0,6-1,5$ & 5811 & Sur \\
\cline { 2 - 8 } & E3 & 22 & S.L. & $1-5$ & $<0,3$ & 5811 & Centro \\
\hline
\end{tabular}


zar libremente en las cuestiones planteadas. Estas preguntas abiertas, a pesar de haber hecho más complejo el análisis, nos han permitido lograr una interpretación mucho más rica y matizada.

Las preguntas se prepararon, agruparon y clasificaron de acuerdo con los objetivos establecidos para el estudio, siguiendo a Josselson (2013), considerando, entre otras, las siguientes recomendaciones: a) predominio de preguntas abiertas frente a cerradas; b) diseñar preguntas que promuevan el discurso narrativo del entrevistado para que la información fluya de un modo natural y orgánico; c) evitar el abuso de preguntas que exijan dar respuesta a un "porqué" y fuercen a un proceso de intelectualización del entrevistado que debería ser realizado por quien realiza la investigación; d) tener muy en cuenta el modo en que el entrevistado se expresa al abordar una cuestión o tema propuesto para valorar mejor su posición al respecto de lo preguntado.

Se agregó un esquema de la cadena del sector, con libros electrónicos como parte de la cadena, para complementar el protocolo. Esto se hizo con el objeto de validarlo y para recopilar información que permita una mejor comprensión de la dinámica entre los enlaces.

Las entrevistas se centraron exclusivamente en las personas que ocupan cargos ejecutivos (presidentes, directores y gerentes). Primero se transcribieron las grabaciones. Después de ser transcritas y consolidadas, se procedió a la lectura de todo el material para organizar y sistematizar las ideas de cara a su posterior análisis (Bardín, 1986). El objetivo de todo este proceso es extraer de las grabaciones un sentido general de la información y evaluar su significado global (Cresswell, 2003).

Se adoptó un enfoque narrativo para transmitir los resultados del análisis, buscando establecer una conexión entre los temas. Esta narrativa fue emparejada con las referencias bibliográficas, buscando una validación de los modelos propuestos en teoría. Este proceso culminó con llegar a las principales implicaciones teóricas y prácticas de esta investigación, descritas en las observaciones finales de este estudio.

\section{ANÁLISIS DE LOS RESULTADOS}

Esta sección trata de responder a las preguntas de investigación planteadas al comienzo del estudio: ¿cuál es el impacto que los libros electrónicos tienen sobre los procesos editoriales? ¿Cómo se configura la cadena de valor del sector editorial español? ¿En qué medida los editores españoles estructuran sus procesos para satisfacer las demandas del consumidor final? Finalmente, se presentan las tendencias latentes en el negocio editorial y se describen los impactos de los cambios tecnológicos en el proceso de publicación y distribución del libro.

\section{Impacto del libro electrónico en los procesos editoriales}

Sobre la base de las entrevistas, se puede establecer, en la figura 5, la configuración probable de la cadena de valor de la publicación, después de la introducción de los libros electrónicos.

En cuanto a las actividades primarias, la llegada del libro electrónico ha ejercido un mayor impacto en el output de la organización que en el input. Las salidas (outputs) toman dos formas: libro impreso y libro electrónico, mientras que las entradas (inputs) se han mantenido igual (textos originales con potencial de mercado).

El desarrollo de contenido sigue siendo la principal fuente de creación de valor en la cadena. Como dijo uno de los entrevistados (E2), "esto solo funciona cuando tienes algo llamado contenido, que es la obra. Si no tienes contenido, no tienes nada".

La producción y distribución física desaparecen dentro del conjunto de las operaciones asociadas al libro electrónico, en favor de las plataformas tecnológicas de comercialización y del marketing digital asociado a la promoción editorial que cuestionan críticamente los modos tradicionales de promoción editorial: el libro electrónico parece vaciar de sentido la idea de presentación y firma de ejemplares a la que el sector estaba acostumbrado con el formato en papel. En tal sentido, todos los responsables entrevistados están de acuerdo en que habrá que buscar nuevas estrategias para promocionar a los autores de narrativa y poesía, principalmente.

Además, la expansión de la capacidad de almacenamiento en servidores (hosts) y el desarrollo de nuevas habilidades en el área de los recursos humanos son decisivos en la transición a la oferta de libros electrónicos. Esta transición generalmente requiere el desarrollo de nuevas habilidades por parte de las organizaciones, así como cambios en la relación entre las empresas (Coreynen, Matthyssens y Bockhaven, 2017; Lin y otros, 2013).

\section{Configuración ampliada de la cadena de valor editorial española}

Hoy en día, la cadena de valor tradicional del sector editorial se ha modificado significativamente, produciéndose una ampliación de aquélla, con la aparición del libro electrónico. Como consecuencia de ello, la editorial puede optar por tres opciones: editar exclusivamente libro en papel, editar exclusivamente libro electrónico y editar en ambos formatos. Las entrevistas con las editoriales permitieron entender cómo son las interacciones con otros sectores cuando se edita libro electrónico tal y como se muestra en la figura 6. 
Figura 5. La cadena de valor genérica de la editorial cuando publica libros electrónicos

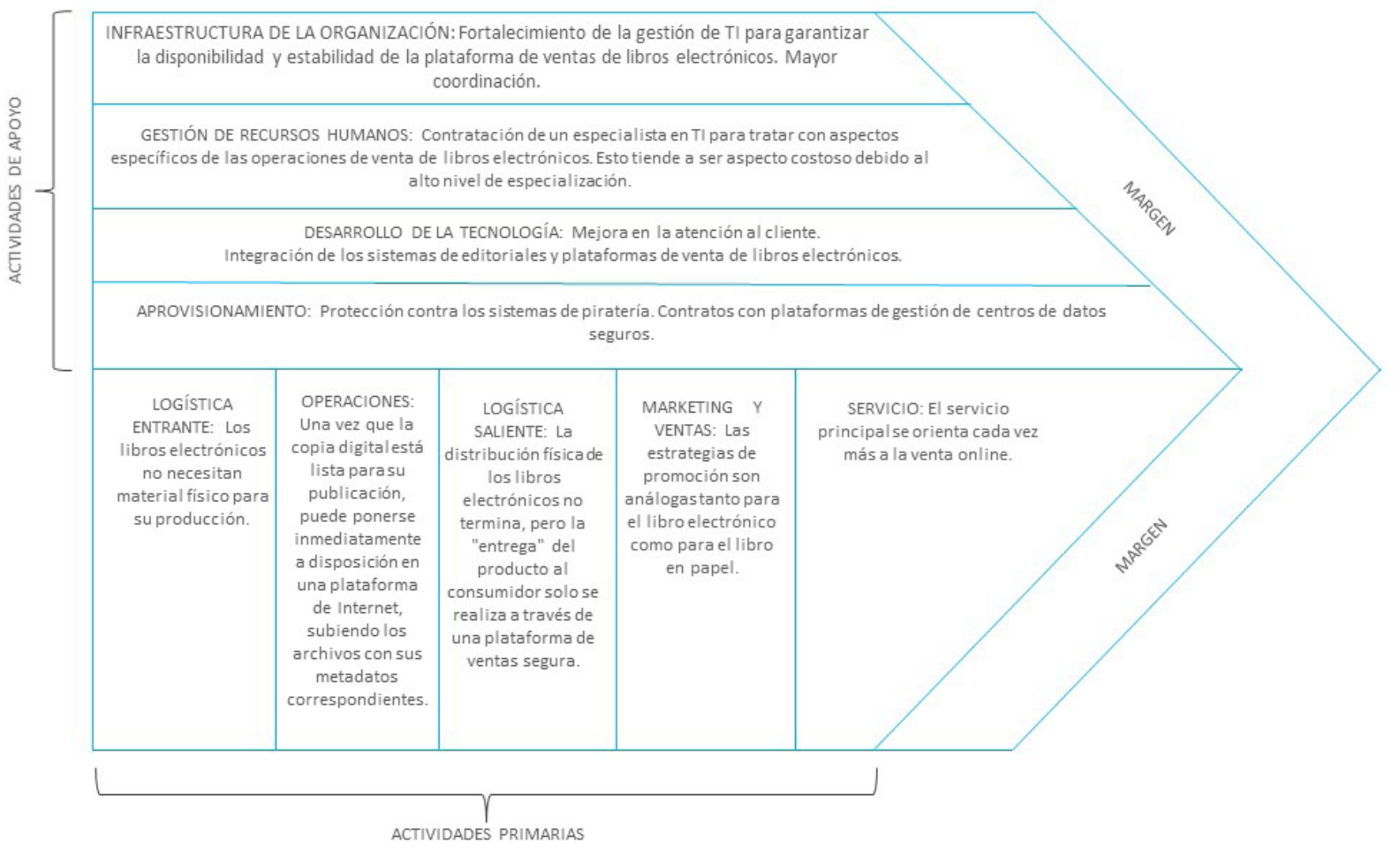

Fuente: Magadán (2017).

Figura 6. Interacción de la empresa editorial (libro electrónico) con otros sectores

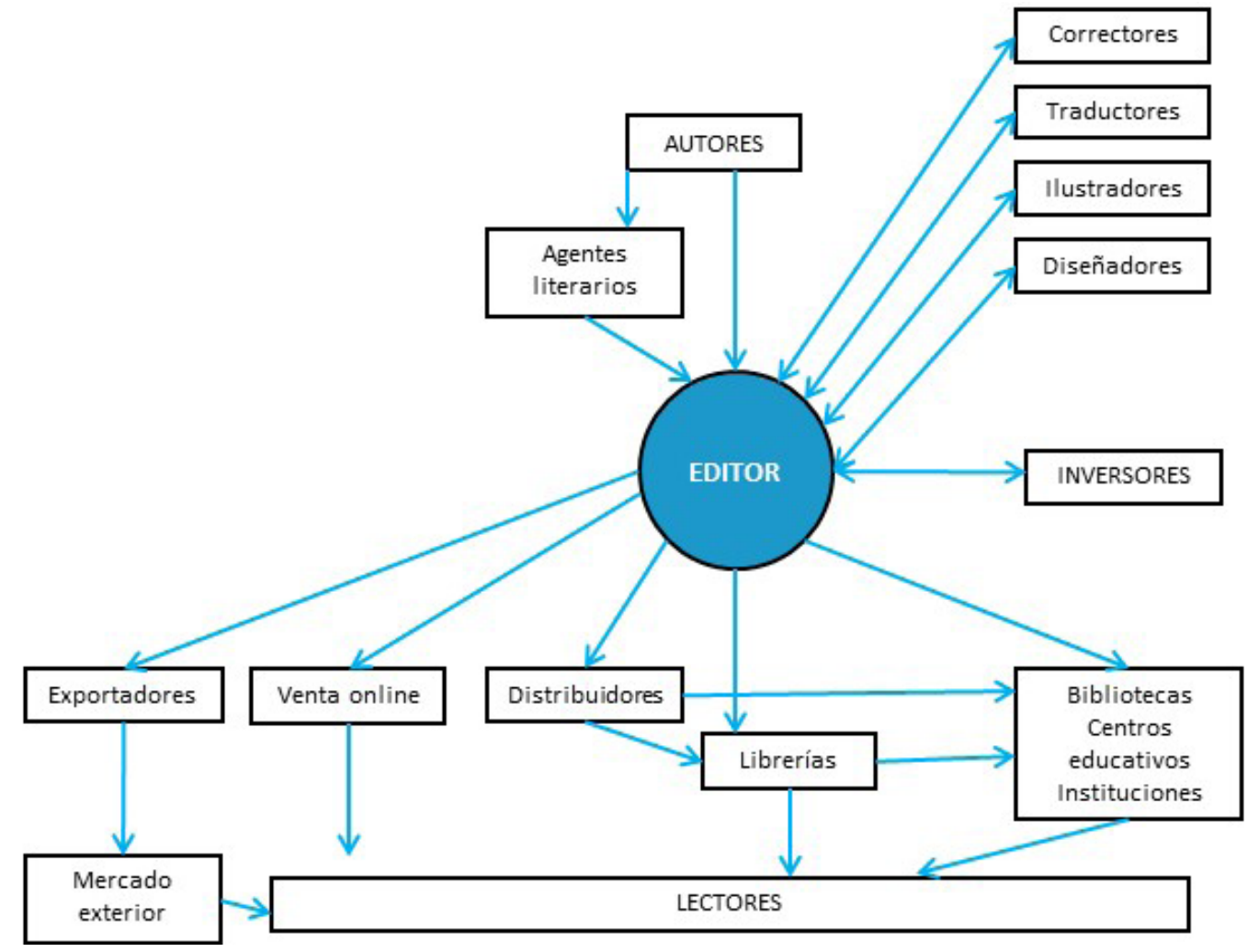


En la figura 7 se genera la cadena de valor obtenida a partir de las entrevistas realizadas con los representantes de cada una de las editoriales y, en ella se muestra la cadena de valor ampliada del sector editorial, donde se observa una superposición entre los flujos de los libros en papel y los libros electrónicos.

El responsable de la editorial E2 considera que la cadena de valor sí ha cambiado con la revolución tecnológica. El más perjudicado de ese cambio va a ser el distribuidor del libro en papel. Para el responsable de E2 es muy difícil hacerse con espacio en las librerías para una editorial como la suya, pero la web y las redes sociales permiten dotarse de visibilidad. En la misma línea se posiciona el responsable de la editorial E3, quien sostiene que la cadena de valor ha cambiado y que los más perjudicados son los distribuidores del libro en papel y que, con el tiempo, se irá hacia un sistema editor-librería. En sentido contrario, se posiciona el responsable de la editorial E1, quien considera que la cadena de valor no va a cambiar en su esencia, aunque las nuevas tecnologías -reconoce- han mejorado la comunicación entre todas las organizaciones que forman parte de ella.

Recurriendo a la información obtenida a través del análisis de casos, encontramos que, en cuanto al libro electrónico, de las tres editoriales analizadas, solo E1 y E2 lo editan y señalan ambas, que, en esta nueva línea de negocio, no ven que se deba mantener el modelo de comercialización tradicional del libro en papel. La editorial E3 no se plantea hacer libros electrónicos: "sería traicionar nuestra filosofía". Desde el punto de vista del responsable de la empresa E2, "el mercado del libro electrónico es residual, con pocas descargas. Tenemos que estar preparados: nuestro fondo se encuentra prácticamente digitalizado" y añade que el sector va avanzando hacia un "modelo comercial donde los intermediarios tenga poca o ninguna presencia".

E1 y E2 comercializan sus libros electrónicos a través de plataformas y distribuidoras digitales. Ninguno de sus responsables se ha planteado integrar en su organización la plataforma y la distribuidora digital. Todos coinciden que la principal barrera es la falta de recursos financieros. El responsable de E2 va más allá y señala que "hacer una buena plataforma exige un equipo de informáticos muy profesionales, que sepan estar a la última en los cambios que se producen. Esto significa tiempo y dinero $y$, de lo último, vamos escasos". Por tanto, la falta de capacidad financiera y conocimientos tecnológicos provocará que, al menos en el corto plazo, las editoriales que publican libro electrónico no integren en su organización la distribución y la venta del libro electrónico. La ausencia de competencias efectivas y de experiencia tecnológica por parte de la editorial (Øiestad y Bugge, 2014) da lugar a que las empresas tecnológicas lideren la comercialización del libro electrónico.

Figura 7. La cadena de valor ampliada del sector editorial

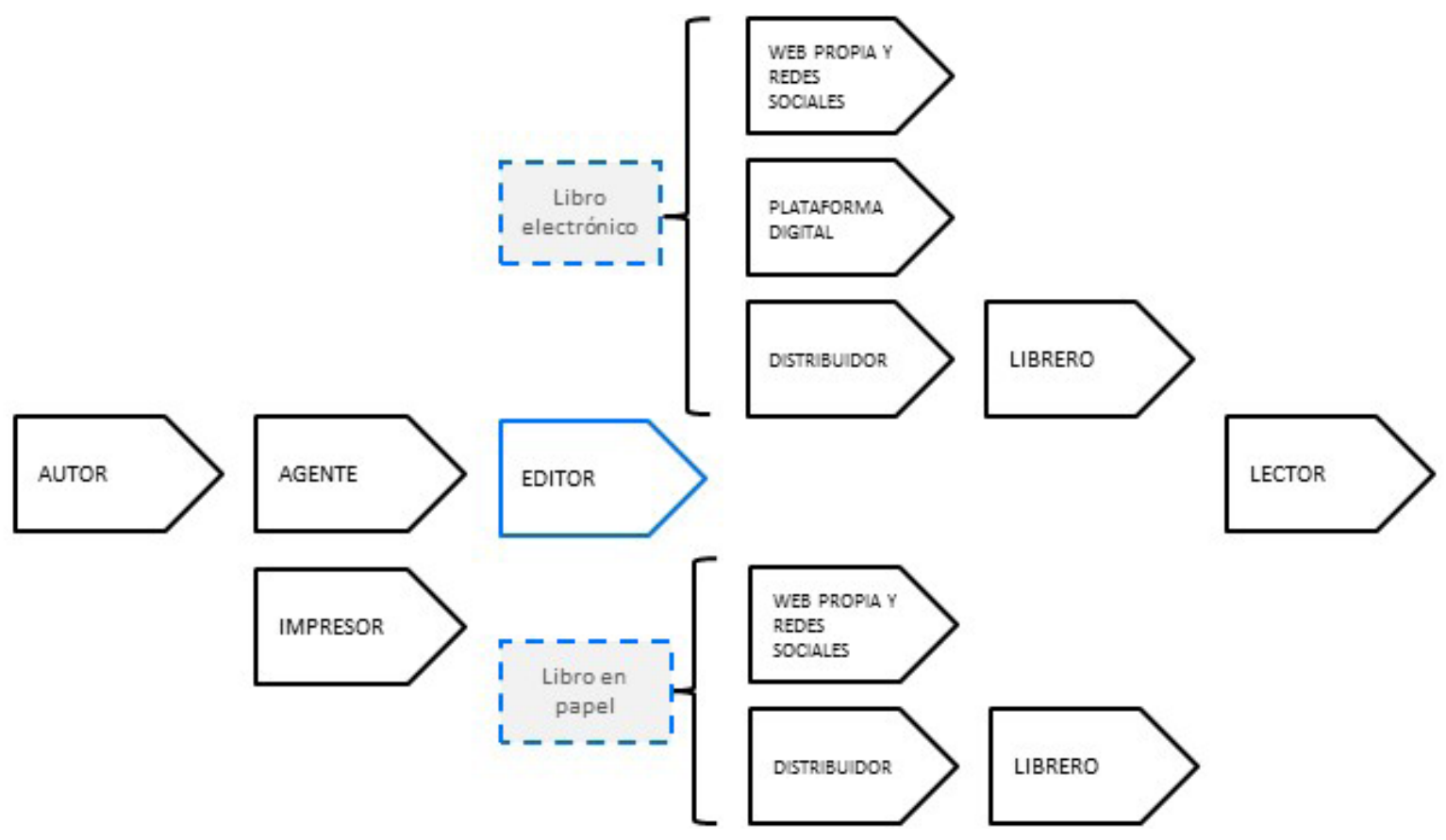


Las editoriales analizadas perciben, en este caso, un menor riesgo en las transacciones de mercado, a pesar de la existencia de altos costes de transacción y el comportamiento oportunista de las plataformas y distribuidoras digitales. Las editoriales consultadas prefieren que sean las plataformas y distribuidoras digitales quienes enlacen su fondo editorial de libros electrónicos con otras plataformas internacionales y librerías.

Al preguntar a los responsables de las editoriales por su visión sobre el fenómeno de la autopublicación asociado al nuevo contexto tecnológico, E1 no la considera una amenaza porque "generalmente muchos de estos autores no tendrían cabida en una editorial con un proceso de evaluación previo y riguroso de su obra". E2 apunta una idea análoga enriquecida con el concepto de "síndrome de autor": "muchos de estos aspirantes a escritores se ven a sí mismos aumentados y no perciben la justa medida de sus capacidades, por eso está muy bien que se metan en faena y afronten el trabajo de editar, aunque solo sea un libro, el suyo, para darse cuenta de la realidad del mercado". Finalmente, E3 considera que si bien "para algunos colegas del sector, la autoedición puede ser una carta de presentación del autor, en mi caso, lo veo como un fenómeno que, si el autor no lo ha asimilado bien, a la larga enturbia la relación con el editor. Personalmente, no edito autores que se hayan autoeditado previamente".

En resumen, podemos concluir que no ha habido una simplificación en la cadena de publicación, ya que los libros electrónicos no reemplazaron a los libros impresos (Carreiro, 2010). En definitiva, los libros electrónicos han creado un nuevo mercado, por lo que la cadena de valor editorial ha tenido que adaptarse para responder a la demanda de ambos formatos.

\section{Adaptación a la demanda final}

Los encuestados de las editoriales E1 y E2 han declarado que se unieron a la producción de libros electrónicos, en parte, debido a la presión de sus autores y del mercado. Sin embargo, los propios editores señalaron que las ventas de libros electrónicos todavía tienen un nivel bajo de facturación.

Las tres editoriales analizadas (E1, E2 y E3) comercializan directamente a través de su web el fondo editorial de libro en papel. La venta de libros a través de la web de la editorial reduce los costes implicados en la distribución y venta del fondo editorial y puede ofrecer al cliente la posibilidad de leer algunos capítulos, además de facilitarle toda la información que sea posible, como, por ejemplo, precio y disponibilidad.
Las tecnologías de la información permiten aumentar la cantidad de información disponible para cada producto y estandarizar los atributos del mismo. En el caso de los libros, la información sobre el producto editorial deberá incluir aspectos tales como el título, el nombre del autor/res, el número de páginas, el precio, el ISBN, las dimensiones del libro, el tipo de encuadernación y la reseña, entre otros. El responsable de la editorial E2 señala que "una venta por Internet supone para nosotros dos ventas por distribuidor". Para el responsable de la editorial E1, "Internet nos ha supuesto un canal más de distribución".

La impresión bajo demanda también está transformando la forma en que los editores ofertan los libros en papel: ya no es necesario imprimir varias unidades de un título (tirada mínima) y esperar a que se vendan, provocando un apalancamiento de recursos materializados en unos ejemplares que generan costes de almacenamiento. Con la impresión bajo demanda la adaptación de la oferta a la demanda podría decirse que es casi perfecta.

Todas las editoriales analizadas están de acuerdo en que la falta de capacidad financiera y conocimientos tecnológicos por parte de las empresas editoriales provocará, al menos en el corto plazo, que sean las empresas tecnológicas con recursos financieros quienes controlen la distribución y venta del libro digital, así como los nuevos modelos que puedan surgir. Para el responsable de la editorial E2, "la amenaza viene de que un único agente, o muy pocos, acaben controlándola". Aunque la editorial E3 no edita libro electrónico, su responsable estima que "empresas como Google o Amazon dan los servicios que las librerías tradicionales renunciaron a prestar: mostrar la práctica totalidad de la oferta disponible y, por tanto, dar visibilidad a las pequeñas editoriales". Para el responsable de la editorial E1, "los editores necesitamos que haya competencia a la hora de distribuir los libros y que no todo se venda a través de estas grandes estructuras".

\section{CONCLUSIONES}

Este estudio tuvo como objetivo evaluar el impacto del libro electrónico sobre la cadena de valor de las editoriales españolas a través del método de estudio de casos, considerando en dicho estudio empresas de diferentes tamaños y siendo conscientes de las limitaciones que esta metodología presenta de cara a la generalización de sus resultados.

Del estudio en profundidad de los tres casos seleccionados en un muestreo por conveniencia, se observó que las principales transformaciones en esta 
cadena están relacionadas con: a) un rápido proceso de desintermediación y b) la redefinición del papel de algunos enlaces, como las librerías físicas.

De la información estadística disponible y las valoraciones de los responsables de las editoriales estudiadas se evidenció que el ritmo de crecimiento del mercado del libro electrónico no es lo suficientemente significativo, en estos momentos, como para causar un cambio radical en la cadena de valor editorial; sin embargo, sí ha requerido ciertos ajustes en las operaciones para integrar los procesos productivos de ambos formatos.

En cuanto a la autopublicación, los análisis sugieren que su impacto es muy limitado, con pocas implicaciones para la dinámica de la cadena de publicación de los editores estudiados.

El desarrollo de nuevos canales de distribución a través de Internet y los libros electrónicos han afectado a la cadena de valor del sector editorial, especialmente a las distribuidoras de libro en papel. Al mismo tiempo, con las nuevas innovaciones de productos editoriales, han surgido nuevos actores que se incorporan al mercado editorial, modificando la cadena de valor y alterando las reglas de juego existentes. La aparición de nuevos parti-

\section{REFERENCIAS}

Alonso, J.; Cordón, J. (2011). El libro electrónico y los DRMs. Anuario ThinkEPI, 5, 249-253

Bardín, L. (1986). Análisis de contenido. Madrid: Akal.

Benghozi, P.; Salvador, E. (2015). Technological innovation and RyD. The disregarded dimension of the creative industries: the case of book publishing. Economia della Cultura, 25(2), 255-268.

Benhamou, F. (2015). Fair use and fair competition for digitized cultural goods: the case of eBooks. Journal of Cultural Economics, 39(2), 123-131. https://doi. org/10.1007/s10824-015-9241-x

Berube, L. (2005). E-books in public libraries: a terminal or termination technology? Interlending and Document Supply, 33(1), 14-18. https://doi. org/10.1108/02641610510582090

Bhatiasevi, V.; Dutot, V. (2014). Creative industries and their role in the creative value chain-a comparative study of SMEs in Canada and Thailand. International Journal of Entrepreneurship and Innovation Management, 18(5-6), 466-480. https://doi.org/10.1504/ IJEIM.2014.064721

Brooks, L.; Fitz, G. (2015). Grey Matter(s): Embracing the Publisher Within. The Foundation Review, 7(2), 3850. https://doi.org/10.9707/1944-5660.1248 cipantes, (por ejemplo, Amazon), en el retailing ha golpeado la rentabilidad de las librerías, además de reducir la necesidad de utilizar, por parte de las empresas editoriales, distribuidores editoriales.

La convivencia interna de los dos modelos -digital y papel- en las compañías editoriales subraya su visión de ambos productos como complementarios en lugar de sustitutivos (Magadán y Rivas, 2018a; Mulholland y Bates, 2014; Zhang y Kudva, 2014).

Finalmente, el nuevo contexto digital abre la puerta al desarrollo de nuevos modelos de negocio. La editorial podría sacar provecho de este nuevo entorno, adoptando las innovaciones surgidas al amparo de las tecnologías de la información y la comunicación con objeto de ampliar su cartera de productos.

La investigación presentada, sin embargo, adolece de ciertas limitaciones, que pasamos a exponer. La primera limitación es la relativa a la naturaleza metodológica del estudio de casos que debería completarse con trabajos posteriores de naturaleza inferencial. Finalmente, en segundo lugar, el estudio está circunscrito a lo que ocurre en el sector editorial español, por lo que sería conveniente ampliar el campo de visión con otros países europeos cuyo sector en su estructura sea similar al español.

Brown, S.; Eisenhardt, K. (1997). The art of continuous change: linking complexity theory and time-paced evolution in relentlessly. Administrative Science Quarterly, 42(1), 1-34.

Caputo, A. (2012). Reflections on the state of specialized libraries: Five global trends all knowledge professionals should understand. Information Services and Use, 32(3-4), 169-171. https://doi.org/10.3233/ISU-20120666

Carreiro, E. (2010). Electronic books: how digital devices and supplementary new technologies are changing the face of the publishing industry. Publishing Research Quarterly, 26, 219-235. https://doi.org/10.1007/ s12109-010-9178-z

Chao, C.; Fuxman, L.; Elifoglu, I. (2013). Electronic books impact global environment-An empirical study focus on user perspectives. Journal of Management and Strategy, 4(2), 52-59. https://doi.org/10.5430/jms. v4n2p52

Chao, C.; Lu, F. (2011). Emergence of e-books and related managerial issues: A preliminary study. International Journal of Business, Marketing, and Decision Sciences, 4(1), 117-126.

Chetty, S. (1996). The case study method for research in small-and medium-sized firms. International Small Business Journal, 15(1), 73-86. 
Corbetta, P. (2003). Metodología y técnicas de investigación social. Madrid: McGraw-Hill Interamericana .

Coreynen, W.; Matthyssens, P.; Bockhaven, W. (2017). Boosting servitization through digitization: Pathways and dynamic resource configurations for manufacturers. Industrial Marketing Management,60, 42-53. https://doi.org/10.1016/j.indmarman.2016.04.012

Cresswell, J. (2003). Research design: Qualitative, quantitative, and mixed methods approaches. Thousand Oaks, Calif: Sage.

Díaz, J. (2010). ¿El año de las tabletas y los e-readers? Dispositivos de lectura para medios de comunicación. Anuario ThinkEPI, 1, 174-179.

Eisenhardt, K. (1989). Building theories from case study research. Academy of Management Review, 14, 532550.

Elman, C.; Gerring, J.; Mahoney, J. (2016). Case study research: putting the quant Into the qual. Sociological Methods and Research, 45(3), 375-391.

FGEE (2007). Comercio interior del libro 2006. Madrid: Federación de Gremios de Editores de España (FGEE).

FGEE (2008). Comercio interior del libro 2007. Madrid: Federación de Gremios de Editores de España (FGEE).

FGEE (2009). Comercio interior del libro 2008. Madrid: Federación de Gremios de Editores de España (FGEE).

FGEE (2010). Comercio interior del libro 2009. Madrid: Federación de Gremios de Editores de España (FGEE).

FGEE (2011). Comercio interior del libro 2010. Madrid: Federación de Gremios de Editores de España (FGEE).

FGEE (2012). Comercio interior del libro 2011. Madrid: Federación de Gremios de Editores de España (FGEE).

FGEE (2014). Comercio interior del libro 2013. Madrid: Federación de Gremios de Editores de España (FGEE).

FGEE (2015). Comercio interior del libro 2014. Madrid: Federación de Gremios de Editores de España (FGEE).

FGEE (2016). Comercio interior del libro 2015. Madrid: Federación de Gremios de Editores de España (FGEE).

FGEE (2017). Comercio interior del libro 2016. Madrid: Federación de Gremio de Editores de España (FGEE).

FGEE (2018). Comercio interior del libro 2017. Madrid: Federación de Gremio de Editores de España (FGEE).

Gaigher, S. (2012). Digital publishing in the South African trade sub-sector: lessons to learn from disruptive technology. Doctoral dissertation, University of Pretoria.

Gerring, J. (2007). Case study research: principles and practice. Cambridge: Cambridge University Press.

Grunow, D. (1995). The research design in organization studies: problems and prospects. Organization science, 6(1), 93-103.

Humphreys, A. (2006). The past, present and future of immersive and extractive ebooks. En P. Messaris; L. Hum- phreys (eds.), Digital media: Transformations in human communication (págs. 159-170). New York: Peter Lang.

Jonas, O.; Sirkeci, I. (2018). Understanding determinants of illegal e-book downloading behaviour in the UK and Germany. Transnational Marketing Journal, 6(2), 79-100.

Josselson, R. (2013). Interviewing for qualitative inquiry: A relational approach. New York, NY: The Guilford Press.

Ketokivi, M.; Choi, T. (2014). Renaissance of case research as a scientific method. Journal of Operations Management, 32(5), 232-240. https://doi.org/10.1016/j. jom.2014.03.004

Lin, C.; Chiou, W.; Huang, S. (2013). The challenges facing e-book publishing industry in Taiwan. Procedia Computer Science, 17, 282-289. https://doi.org/10.1016/j.procs.2013.05.037

Longhi, C.; Rochhia, S. (2014). Ceci tuera cela»? Dynamique des changements dans l'industrie du livre. Revue d'économie industrielle, 1, 121-154.

Magadán, M. (2017). Technological change and innovation in the Spanish publishing industry: organizational effects. Oviedo: Doctoral dissertation, University of Oviedo. Obtenido de http://hdl.handle.net/10651/44590

Magadán, M.; Rivas, J. (2018a). Digitization and business models in the Spanish publishing industry. Publishing Research Quarterly, 34(3), 333-346. https://doi. org/10.1007/s12109-018-9593-0

Magadán, M.; Rivas, J. (2018b). The Impact of Digitization on the Spanish publishing industry. The International Journal of the Book, 16(2), 1-18. https://doi. org/10.18848/1447-9516/CGP/v16i02/1-18

Magadán, M.; Rivas, J. (2019). Fiscalidad indirecta del libro en Europa: implicaciones y políticas. El profesional de la información, 28(1), e280111. https://doi. org//10.3145/epi.2019.ene.11

Marszałek, P. (2016). Disintermediation of banks: Causes and consequences. Research Papers of the Wroclaw University of Economics, 451, 256-267.

Matulionyte, R.; Paton, E.; McIntyre, P.; Gleadhill, D. (2017). The system of book creation: Intellectual property and the selfpublishing sector of the creative industries. Creative Industries Journal, 10(3), 191-210. https://doi.org/10.1080/17510694.2017.1393193

McCutcheon, D. M., y Meredith, J. R. (1993). Conducting case study research in operations management. Journal of Operations Management, 11(3), 239-256. https://doi.org/10.1016/0272-6963(93)90002-7

Miles, M.; Huberman, A. M.; Saldaña, J. (2013). Qualitative data analysis: A methods sourcebook. Thousand Oaks, CA: Sage.

Mintzberg, H. (1973). The nature of managerial work. New York: Harper and Row.

Mulholland, E.; Bates, J. (2014). Use and perceptions of e-books by academic staff in further education. The Journal of Academic Librarianship, 40(5), 492-499. http://doi.org/10.1016/j.acalib.2014.05.018 
Observatorio de la Lectura y el Libro. (2018). Panorámica de la edición española de libros 2017. Análisis sectorial del libro. Madrid: Secretaría General Técnica. https:// sede.educacion.gob.es/publiventa/panoramica-de-laedicion-espanola-de-libros-2017-analisis-sectorial-dellibro/edicion/21499C [Fecha de consulta: 11/10/2018].

Øiestad, S.; Bugge, M. (2014). Digitisation of publishing: exploration based on existing business models. Technological Forecasting and Social Change, 83, 54-65. https://doi.org/10.1016/j.techfore.2013.01.010

Orosa, B. G.; García, X. (2016). El ebook busca en la lectura social la propuesta que impulse nuevos formatos de éxito. Anales de Documentación, 19(2), 1-7. https://doi.org/10.6018/analesdoc.19.2.246351

Peng, Y. (2016). Mobile and digitally-mediated publishing strategies in China: an overview of evolving business models. Publishing Research Quarterly, 32(3), 247260. https://doi.org/10.1007/s12109-016-9467-2

Pettigrew, A. M.; Whipp, R. (1991). Managing change for competitive success. Oxford: Blackwell.

Rao, S. S. (2005). Electronic books: their integration into library and information centers. The Electronic Library, 23, 116-140. https://doi. org/10.1108/02640470510582790

Sánchez, P. D.; Torres, Y.; Casaubón, M.; Puig, C.; Gràcia, J. (2011). Desarrollo del servicio de préstamo en línea de libros electrónicos en las bibliotecas públicas de Cataluña y de la Comunidad de Madrid. BiD: Textos Universitaris de Biblioteconomia i Documentació, 26, 3. http://bid.ub.edu/26/pdf/vives2.pdf
Shatzkin, M. (2008). Digital publishing in the US: driving the industry to vertical niches? Logos, 19(2), 56-60.

Simons, H. (2011). El estudio de caso. Teoría y práctica. México: Ediciones Morata.

Snow, C.; Thomas, J. (1994). Field research methods in strategic management: contributions to theory building and testing. Journal of Management Studies, 31(4), 457-480.

Van de Pen, A. H.; Poole, M. S. (1990). Methods to develop a grounded theory of innovation processes in the Minnesota innovation research program. Organization Science, 1, 315-335.

Vasileiou, M.; Rowley, J. (2008). Progressing the definition of "e-book". Library Hi Tech, 26(3), 355-368. https://doi.org/10.1108/07378830810903292

Wilson, E. J.; Vlosky, R. (1997). Partnering relationship activities: building theory from case study research. Journal of Business Research, 39(1), 59-79.

Yin, R. (2009). Case study research. Design and methods (4a ed.). London: Sage Publications.

Yin, R. (2011). Applications of case study research (3a ed.). London: Sage Publications.

Zhang, Y.; Kudva, S. (2014). E-books versus print books: Readers' choices and preferences across contexts. Journal of the Association for Information Science and Technology, 65(8), 1695-1706. https://doi. org/10.1002/asi.23076 